\title{
Novel fusion electrokinetic technology with gas re-injection (GREK) for soil remediation and stabilization
}

\author{
Sanghee Shin \\ Geotechnical Engineering Research Division, Korea Institute of Construction and Technology, Goyang-Si, Gyeonggi-Do, Republic of \\ Korea
}

\author{
Email address: \\ scott@kict.re.kr
}

\section{To cite this article:}

Sanghee Shin. Novel Fusion Electrokinetic Technology with Gas Re-Injection (GREK) for Soil Remediation and Stabilization. Earth Science. Vol. 3, No. 1, 2014, pp. 9-13. doi: 10.11648/j.earth.20140301.12

\begin{abstract}
In this study, a gas reinjection type electrokinetic remediation treatment process (GREK) was proposed as the method for the restoration and stabilization of contaminated soil. The proposed electrokinetic remediation was a new technique, where treatment was performed by reinjecting the surplus gas, which was produced from an anode chamber, into soil, in order to remove the heavy metals and moisture that exist in clayey soil or clayey-sandy soil with low hydraulic conductivity. The manufactured samples were completed through the compression process, in which pressure was gradually increased to a final pressure of $2.11 \mathrm{kgf} / \mathrm{cm}^{2}$ (30 psi) during a total of 7 days. Before the compression, the samples were taken, and the heavy metal concentration and water content were measured. For the gas reinjection type electrokinetic remediation treatment process (GREK) and non-injection type treatment method, continuous treatment processes were performed for 24 hours at a voltage gradient of $2 \mathrm{~V} / \mathrm{cm}$. The results of the water content indicated that the gas reinjection type electrokinetic remediation treatment process (GREK) was about 3\% higher than that for non-injection type treatment method. The removal efficiency of chromium $(\mathrm{Cr})$ indicated that the gas reinjection type electrokinetic remediation treatment process (GREK) was about 15\% higher than that for non-injection type treatment method. The removal efficiency of cadmium $(\mathrm{Cd})$ indicated that the gas reinjection type electrokinetic remediation treatment process (GREK) was about 22\% higher than that for non-injection type treatment method. It is expected that the proposed gas reinjection type treatment method would contribute to convergence electrokinetic treatment processes in the future.
\end{abstract}

Keyword: Gas Injection Electrokinetic, Electroremediation, Clay, Clay-Sandy Soil, Gas Control Process

\section{Introduction}

Electrokinetic remediation, which was used for the restoration and stabilization of contaminated soil, has been performed by various researchers [1-8].Casagrande showed the possibility of dewatering using electrokinetics for soils with diverse characteristics, and performed a study that applied this method [1].In the electrokinetic remediation suggested by Tchilingarian, clay particles with different sizes have different electrophoresis velocities, and using this characteristic, the possibility of the recovery of oil or heavy metal ions present in clay or a rock mass was suggested [2]. This theory has been applied as a field of dewatering process in geotechnical engineering and environmental engineering. Ambah and Chilin gar performed convergence research that combined the studies of enhanced oil recovery, which was from oil development and recovery field, with the geotechnical engineering field
$[3,9,10,11]$.Especially, for the use of electrical enhanced oil recovery, various experiments were performed by constructing a low-power mechanism, and each characteristic was analyzed[9, 10, 11].In addition, Pamukcu suggested a restoration method for the industrial area contaminated with oil, studied the electrokinetic removal of hydrocarbons from clayey soil, and evaluated the removal efficiency of hydrocarbons $[4,5]$.Theenhanced oil recovery method using electrokinetic remediation can recover oil at a low cost since it does not require additional subsidiary facilities, and was one of the important techniques that can remove ionic materials from a medium with low hydraulic conductivity (e.g., clay).This technique consumes less water compared to the existing oil recovery method which was used along with a steam injector, and also does not require additional water injection. The enhanced oil recovery method using electrokinetic remediation can minimize equipment on the ground surface, and does not 
require subsidiary facilities such as steam generator, compressor, fluid pump, and harmful material storage tank. Field-scale experiments have been successfully carried out in California, USA and in Canada [12].Shin performed a study where acidic solution was additionally injected into the anode while using electrokinetic remediation in order to improve the efficiency of this process [12, 13].Unlike general electrokinetic remediation processes, the inserted process was a method in which the resource recovery rate was increased by increasing the flow velocity and flux, through injecting diluted hydrochloric acid solution using a pump and through enlarging the cracks of a rock mass with low hydraulic conductivity $[8,12,13]$.

The purposes of this study were to investigate the possibility of the restoration and stabilization of contaminated soil using the surplus gas, which was produced during the treatment of high-salinity $(40,000 \mathrm{ppm})$ near shore clayey-sandy soil using electrokinetic remediation, and to examine the differences in the efficiency between the cases of the gas reinjection type electrokinetic remediation treatment process (GREK) and non-injection type treatment method.

\section{Theoretical Background}

The physicochemical reaction process of general electrokinetic remediation was progressed by the direct current which was determined by the voltage ratio. $\mathrm{H}^{+}$and $\mathrm{O}_{2}$ gas were produced at the anode; and $\mathrm{OH}^{-}$and $\mathrm{H}_{2}$ gas were produced at the cathode.

$$
\begin{aligned}
& \mathrm{H}_{2} \mathrm{O}-2 \mathrm{e}^{-} \rightarrow 1 / 2 \mathrm{O}_{2}+2 \mathrm{H}^{+} \\
& 2 \mathrm{H}_{2} \mathrm{O}+2 \mathrm{e}^{-} \rightarrow \mathrm{H}_{2}+2 \mathrm{OH}
\end{aligned}
$$

Equations 1 and 2 show the chemical compositions that were progressed at the anode and the cathode after the electrolysis of the water. Typical seawater has a salinity of $35,000 \mathrm{ppm}$. However, for high-salinity seawater with a salinity of $40,000 \mathrm{ppm}, \mathrm{Cl}_{2}$ gas was additionally produced at the anode, where $\mathrm{H}^{+}$and $\mathrm{O}_{2}$ gas were produced, as shown in Equation 3.

$$
\begin{aligned}
& \mathrm{H}_{2} \mathrm{O}-2 \mathrm{e}^{-} \rightarrow 1 / 2 \mathrm{O}_{2}+2 \mathrm{H}^{+} \\
& 2 \mathrm{Cl}^{-}-2 \mathrm{e}^{-} \rightarrow \mathrm{Cl}_{2}
\end{aligned}
$$

Fig. 1 shows the moving direction of fluidized materials (e.g., oil) or ionic materials, when electrokinetic remediation was applied to rock or a clayey medium with low hydraulic conductivity. At the center (III), the flow of the fluid progresses from the anode to the cathode; and the surface and interior of the rock or the clayey medium with low hydraulic conductivity were represented by the fluidized double layers (II) and the non-fluidized layer (I), respectively.

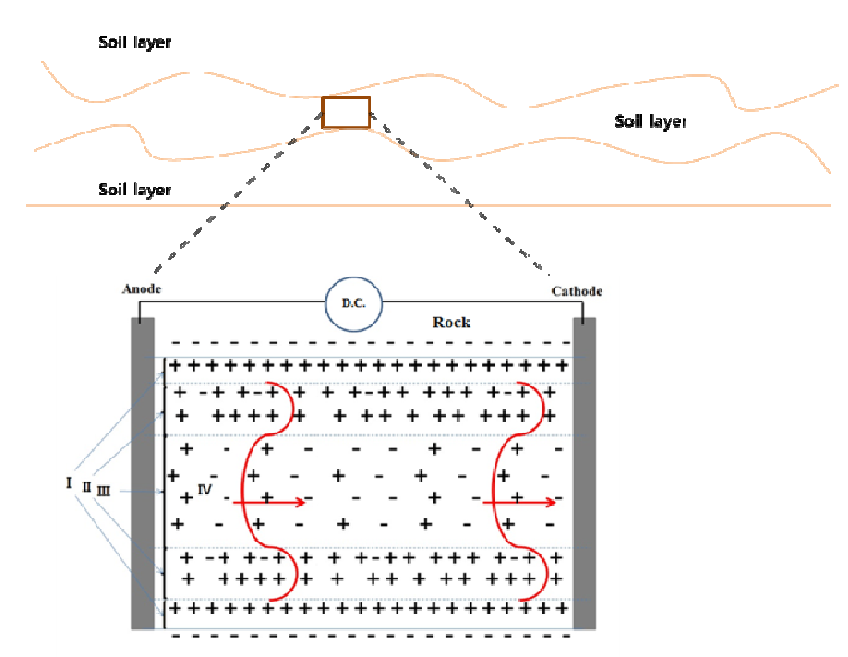

Fig 1.Schematic diagram of electrokinetic double layer; I: Immobile double layer, II: Mobile double layer, III: Free water, IV: Velocity profile (solid curved line - velocity profile in a capillary). Rock was negatively charged [13, 14].

\section{Experiment Configuration and Results}

\subsection{Experiment Configuration}

For the samples used in the experiment, the clayey-sandy soil of an oil refinery, which was located near Abu Dhabi, the capital of UAE, was used. As the sampling area was near the oil refining facility, the area had been already contaminated, and clams had already died. For this reason, large solid materials such as clamshells were included in the collected samples. Thus, the samples for the experiment were manufactured after removing the solid materials using a10 $\mathrm{mm}$ sieve. The samples used in the experiment were manufactured to be a length of $30 \mathrm{~cm}$ and a diameter of $3.81 \mathrm{~cm}$. To examine the metal material extraction capacity, the samples were compressed by gradually increasing pressure to a final pressure of $2.11 \mathrm{~kg} \mathrm{f} / \mathrm{cm}^{2}$ (30 psi)during 7 days. The numbers shown in Fig. 2 represent the sampling spots, and the flow of the current progresses from the anode to the cathode.

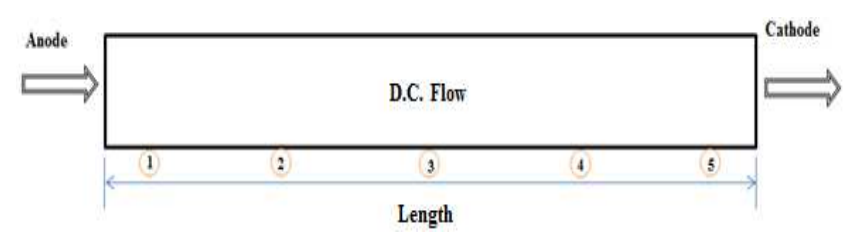

Fig 2.Schematic diagram for the direct current (D.C.) flow directionat sample. 


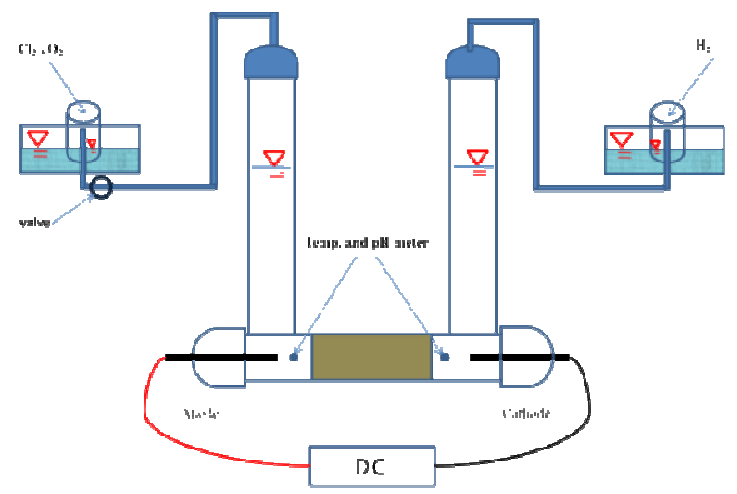

Fig 3.Apparatus used in soil remediation and stabilization of clay component contaminated with heavy metals from offshore sediments [13, $14]$.

The water contents before and after the experiment were measured to examine the amount of fluid extraction. Based on the constructed experiment model shown in Fig. 3, the experiment was performed for 24 hours at a voltage gradient of $2 \mathrm{~V} / \mathrm{cm}$. Two experiments were conducted by dividing them into gas reinjection type electrokinetic remediation treatment process (GREK) and non-injection type treatment method.

In the case of gas reinjection type electrokinetic remediation treatment process (GREK), gas was reinjected into the samples by sealing the gas outlet installed at the anode; and in the case of non-injection type treatment method, exhaust gas was discharged after the stabilization in a safe collection facility.

\subsection{Experiment Results}

The results of the treatment of the soils with low hydraulic conductivity (e.g., clayey soil or clayey-sandy soil) using the gas reinjection type electrokinetic remediation treatment process (GREK) and non-injection type treatment method were presented below. The graph in Fig. 4 shows the measurement results of the water contents before and after the experiment for the two experiments performed in this study.

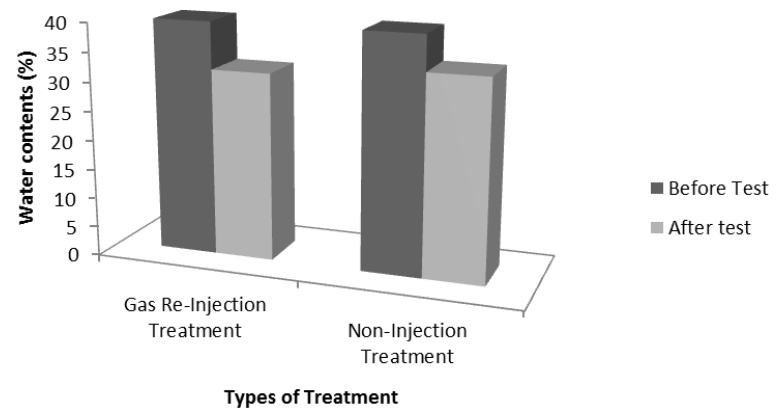

Fig 4.Comparison between Gas re-injection treatment and non-injection treatment with water contents

The weights of the prepared samples were about $1026 \mathrm{~g}$ for the case of the gas reinjection type electrokinetic remediation treatment process (GREK), and about $1029 \mathrm{~g}$ for the case of non-injection type treatment method; and the initial water contents were measured to be $40 \%$, respectively. When the water contents were measured after the 24-hour treatment, the case of the gas reinjection type electrokinetic remediation treatment process (GREK) was $31 \%$, and the case of non-injection type treatment method was $34 \%$.The results of the experiment indicated that the water content gas reinjection type electrokinetic remediation treatment process (GREK) was about 3\% higher than that for non-injection type treatment method.

To examine the changes in the concentration within the samples, the samples were taken before and after the experiment, and the concentrations were measured using ICP-MS. In the case of the heavy metals for measurement, they were limited to $\mathrm{Cr}$ and $\mathrm{Cd}$ because natural samples were analyzed. The initial $\mathrm{Cr}$ concentrations were 29.83 ppb for the case of gas reinjection type electrokinetic remediation treatment process (GREK), and $25.34 \mathrm{ppb}$ for the case of non-injection type treatment method. In the case of the concentrations after the 24-hour treatment, the measurements were made by collecting the samples from the spots marked with the numbers shown in Fig. 2.Spot 5 showed the highest treatment ratio, and the concentrations were $19.72 \mathrm{ppb}$ and $20.76 \mathrm{ppb}$. Also, the treatment efficiencies were $34 \%$ for the case of gas reinjection type electrokinetic remediation treatment process (GREK), and $18 \%$ for the case of non-injection type treatment method. The initial Cd concentrations were $0.146 \mathrm{ppb}$ for the case of the gas reinjection type electrokinetic remediation treatment process (GREK), and $0.080 \mathrm{ppb}$ for the case of noninjection type treatment method. In the case of the concentrations after the 24-hour treatment, the measurements were made by collecting the samples from the spots marked with the numbers shown in Fig 2. Spot 5 showed the highest treatment ratio, and the concentrations were $0.074 \mathrm{ppb}$ and $0.059 \mathrm{ppb}$. Also, the treatment efficiencies were $49 \%$ for the case of the gas reinjection type electrokinetic remediation treatment process (GREK), and $27 \%$ for the case of non-injection type treatment method. The pressure head that corresponded to the amount of the gas produced by the electrokinetic reaction was included in the hydraulic pressure head value. As a result, in each sample, the total velocity and total flux due to the electrokinetic hydraulic head increased. The graphs in Figs. 5 and 6 show the $\mathrm{Cr}$ and $\mathrm{Cd}$ concentrations before and after the treatment.

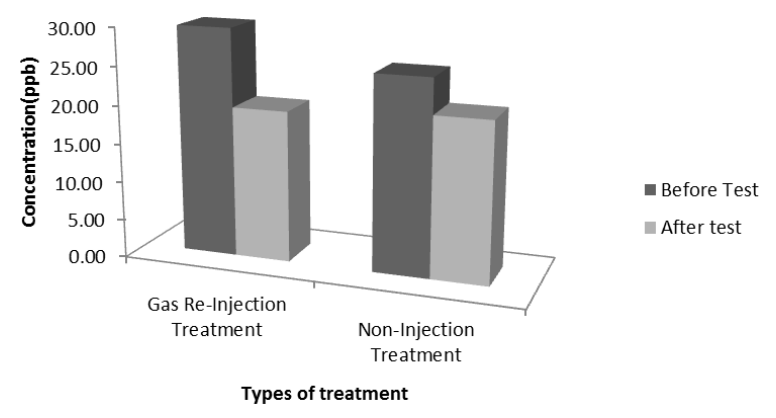

Fig 5. Cr Concentration of gas re-injection treatment vs. non-injection treatment after 24 hours treatment with $2 \mathrm{~V} / \mathrm{cm}$ 


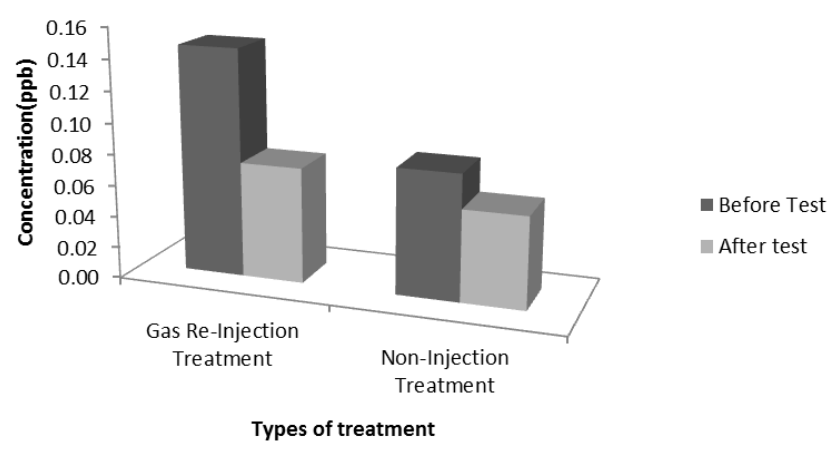

Fig 6.Cd Concentration of gas re-injection treatment vs. non-injection treatment after 24 hours treatment with $2 \mathrm{~V} / \mathrm{cm}$

In Fig. 2, the $\mathrm{pH}$ of the sample from spot 1 was maintained at $6.8 \sim 6.9$, and the $\mathrm{pH}$ of the sample from spot 5 was maintained at $8.4 \sim 8.6$. Therefore, the changes in the $\mathrm{pH}$ could be examined depending on the flow of the electrokinetic remediation.

In Fig. 7, the initial $\mathrm{pH}$ for the gas reinjection type treatment method was 7.81. After the experiment, the $\mathrm{pH}$ was 6.8 at sampling spot 1 near the anode, and the $\mathrm{pH}$ was 8.24 at sampling spot 5 near the cathode. The initial $\mathrm{pH}$ for the non-injection type treatment method was 7.43. After the experiment, the $\mathrm{pH}$ was 6.95 at sampling spot 1 near the anode, and the $\mathrm{pH}$ was 8.45 at sampling spot 5 near the cathode.

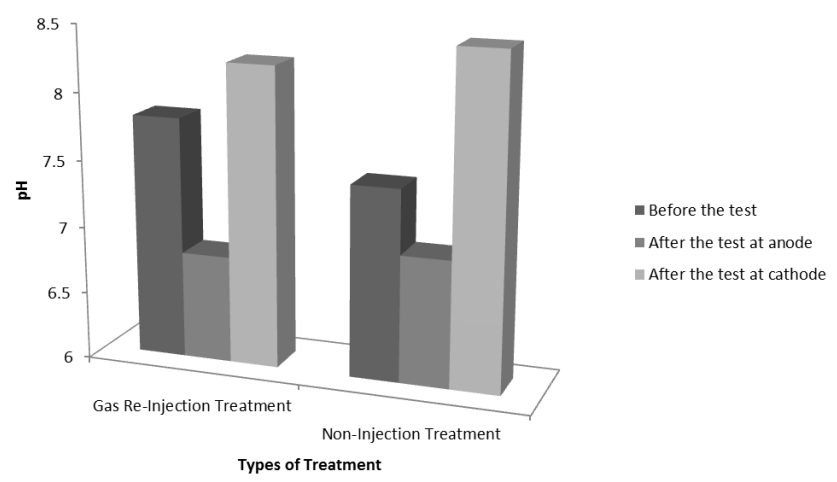

Fig 7.Comparison sample the initial $p H$ vs. the spot $5 \mathrm{pH}$ at gas reinjection treatment after 24 hours treatment with $2 \mathrm{~V} / \mathrm{cm}$ and Comparison sample the initial $\mathrm{pH}$ vs. the spot $5 \mathrm{pH}$ at non-injection treatment after 24 hours treatment with $2 \mathrm{~V} / \mathrm{cm}$

\section{Conclusion}

The gas reinjection type electrokinetic remediation treatment process (GREK) ashy brid treatment technique was proposed in which the gas produced from the anode was re-injected into samples, was combined with the existing traditional electrokinetic remediation. This technique showed the possibility of the restoration and stabilization of the contaminated clayey soil or clayeysandy soil. It was found that the gas reinjection type electrokinetic remediation treatment process (GREK) improved the decreasing water content and the efficiency of the heavy metal concentration within the samples after the treatment. The changes in the heavy metal concentration within the samples and the water content were used to evaluate the proposed treatment method. The measurement of the water content after 24 hours indicated that the water content the gas reinjection type electrokinetic remediation treatment process (GREK) method was about 3\% higher than that for non-injection type treatment method. The removal efficiency of chromium $(\mathrm{Cr})$ and cadmium (Cd)indicated that the gas reinjection type electrokinetic remediation treatment process (GREK) was about 15\%, 22\% higher than that for non-injection type treatment method, respectively. The flow velocities for each sample calculated using the water content were $0.20 \mathrm{~m} /$ day for the gas reinjection type treatment method, and $0.14 \mathrm{~m} /$ day for the non-injection type treatment method. Also, an additional pressure device was not required because produced gas was reused as pressure energy, and the case of the gas reinjection type electrokinetic remediation treatment process (GREK) showed more efficient performance compared to the case of non-injection type treatment method. Based on the comparison of the measured heavy metal concentrations for the cases of the gas reinjection type electrokinetic remediation treatment process (GREK) and non-injection type treatment method, it was thought that the heavy metals and moisture could be more efficiently removed in the case of the gas reinjection type electrokinetic remediation treatment process (GREK) at the same time period. In short, the water content changed due to the changes in the flow velocity depending on the pressure condition ata specific time period at the same condition, and based on this change, the removal rate of the ionic materials within the samples could be controlled. The $\mathrm{pH}$ values within the samples after the experiment measured in this study were evenly distributed from weakly acidic to weakly alkaline. Therefore, it was expected that this study, which was performed in near shore area, could help minimize the damage of soil environments. In addition, it was environment-friendly because all the processes were performed using an in-situ method.

\section{References}

[1] Casagrande, I. L., "Full scale experiment to increase bearing capacity of piles by electrochemical treatment",Bautechnique, Vol. 15, No.1, 1937, pp. 14-16.

[2] Tchilingarian, G. V., "Possible utilization of electrophoretic phenomenon for separation of fine sediments into grades", Journal of Sedimentary Petrology, Vol. 22, No. 1, 1952, pp. 29-32.

[3] Ambah, S. A., Chilingar, G. V. and Beeson, C. M., "Use of direct electrical current for increasing the flow rate of reservoir fluids during petroleum recovery", Journal of Canadian Petroleum Technology, Vol. 3, No.1, 1964, pp.814.

[4] Pamukcu, S., Weeks, A. and Wittle, J.K., "Electrochemical extraction and stabilization of selected inorganic species in porous media", Journal of Hazardous Materials, Vol. 55, No. 1-3, 1997, pp. 305- 318. 
[5] Pamukcu, S., Weeks, A. andWittle, J.K., "Enhanced reduction of $\mathrm{Cr}$ (VI) by direct electrical current in a contaminated clay", Environmental Science Technology, Vol.38, No. 4, 2004, pp. 1236 - 1241.

[6] M.M. Page, C.L. Page, "Electroremediation of contaminated soils", Journal of Environmental Engineering, Vol.128, 2002, pp. 208-219.

[7] K.J. Kim, D.H. Kim, J.C. Yoo, K. Baek, "Electrokinetic extraction of heavy metals from dredged marine sediment", Separation Science and Technology, Vol.79, No.2, 2011, pp. 164-169.

[8] Chilingar, G. V., Adamson, L. G., Armstrong, R. A. and Beeson, C. M., "Soils stabilized through electroosmosis", Southwest Builder and Contractor, Vol.145, No. 24, 1964, pp.100-102.

[9] Chilingar, G. V., Amba, S. A. and Beeson, C. M., "Application of electrokinetic phenomena in civil engineering and petroleum engineering", Annals of the New York Academy of Science, Vol.118, No. 14, 1965, pp. 585602
[10] Chilingar, G. V., Adamson, L. G., Rieke, H. H. and Gray, R. R., "Electrochemical treatment of shrinking soils", Engineering Geology, Vol. 2, No.3, 1968, pp.197-203.

[11] Wittle, J.K., Hill, D.G. andChilingar, G.V., "Direct Electric Current Oil Recovery (EEOR) - A New Approach to Enhancing Oil Production", Energy Sources, Part A: Recovery, Utilization, and Environmental Effects, Vol. 33, No. 9, 2011, pp. $805-822$.

[12] Shin,S. H., Chilingar, G.V., Haroun, M., Wittle, J.K.,Meshkati, N., Pamukcu, S., Jeoung, J. H. and Koo, H.B. "Electrokinetics Technology to Improve Acidizing of Carbonate Reservoir Rocks",Journal of Environmental Protection, Vol. 4 , No. 4A, 2013, pp. 1-3.

[13] Shin, S.H., Chilingar, G.V. ,Haroun, M., Ghosh, B., Meshkati, N., Pamukcu, S., Wittle, J.K. andBadawi, M.A., "The Effect of Generated Chlorine Gas on Electroremediation of Heavy Metals from Offshore Muds", Journal of Environmental Protection, Vol. 3 , No. 5, . 2012, pp. 363-373. 